\title{
Review on Order Statistics and Record Values from $F^{\alpha}$ Distributions
}

\author{
M. Shakil \\ Department of Mathematics \\ Miami Dade College, Hialeah Campus \\ Hialeah, USA \\ mshakil@mdc.edu \\ M. Ahsanullah \\ Department of Management Sciences \\ Rider University \\ Lawrenceville, USA \\ ahsan@rider.edu
}

\begin{abstract}
Both order statistics andrecords arise naturally in many fields of studies such as climatology, sports, science, engineering, medicine, traffic, and industry, among others. Study of their properties and applications plays important roles in many areas of statistical research, for example, statistical inference, nonparametric statistics, among others. In recent years, the $F^{\alpha}$ distributions have been widely studied in statistics because of their wide applicability in the modeling and analysis of life time data. An absolutely continuous positive random variable $X$ is said to have an $F^{\alpha}$ distribution if its cumulative distribution function is given by $G(x)=F^{\alpha}(x)=[F(x)]^{\alpha}, \alpha>0, x>0$, which is the $\alpha$ th power of the base line distribution function $F(x)$. Many researchers and authors have developed various classes of $F^{\alpha}$ distributions. It appears from the literature that not much attention has been paid to the analysis of record values and order statistics from these members of $F^{\alpha}$ family of distributions, and, therefore, need further and special investigations. In this paper, some distributional properties of order statistics and record values from $F^{\alpha}$ distributions are reviewed. We hope that the findings of this paper will be useful for the practitioners in various fields of studies and further enhancement of research in $F^{\alpha}$ distributions, order statistics, record value theory and their applications.
\end{abstract}

Keywords\& Phrases: Exponentiated distribution, $F^{\alpha}$ distribution, Order statistics, Record values.

2000Mathematics Subject Classifications:60E05, 62E10, 62E15, $62 \mathrm{G} 30$.

\section{Introduction}

Random variables or functions of random variables when put in order from the smallest to the largest are called order statistics. On the other hand, an observation is called a record if its value is greater than (or analogously, less than) all the preceding observations. Both order statistics andrecords arise naturally in many fields of studies such as climatology, sports, science, engineering, medicine, traffic, and industry, among others. As such study of their 
properties and applications play important roles in many areas of statistical research, for example, statistical inference, nonparametric statistics, among others. In recent years, there has been a great interest in $F^{\alpha}$ distributions. An absolutely continuous positive random variable $X$ is said to have $F^{\alpha}$ distribution if its cdf is given by $G(x)=F^{\alpha}(x)=[F(x)]^{\alpha}, \quad \alpha>0, x>0$, which is the $\alpha$ th power of the base line distribution function $F(x)$. The distribution $G(x)$ is also called an exponentiated distribution of the given cumulative distribution function (cdf) $F(x)$. The $F^{\alpha}$ distributions have been widely studied in statistics since 1995 because of their wide applicability in the modeling and analysis of life time data. Many researchers and authors have developed various classes of $F^{\alpha}$ distributions. For example, the exponentiated exponential distribution (EED) or generalized exponential distribution (GED) was introduced by Gupta et al. (1998) as a generalization of the standard exponential distribution to model failure time data by Lehman alternatives, and later studied by Gupta and Kundu (1999, 2000, 2001, 2007). For further developments and distributional properties of the EE distribution, the interested readers are referred to the recent survey paper of Nadarajah (2011). Besides the EED (or GED), several other members of $F^{\alpha}$ family of distributions have been developed by various authors. It appears from the literature that not much attention has been paid to the analysis of record values and order statistics from these members of $F^{\alpha}$ family of distributions, and, therefore, need further and special investigation. In this paper, some distributional properties of order statistics and record values from $F^{\alpha}$ distributions are reviewed. The organization of this paper is as follows. In Section 2 , the exact distributions of order statistics and record values are provided. Section 3 contains the distributional properties of order statistics and record values when the parent distributions are $F^{\alpha}$ type distributions. In Section 4, we briefly state some other members of the $F^{\alpha}$ family of distributions which have recently been developed by various authors. The concluding remarks are presented in Section 5.

\section{The Exact Distributions of Order Statistics and Record Values}

Here we provide the exact distributions of order statistics and record values.

\subsection{Order Statistics: Definitions and Notations}

Suppose that $\left(X_{r}\right),(r=1,2, \ldots, n)$, is a sequence of $n$ independent and identically distributed (i.i.d.) random variables $\left(r v^{\prime} s\right)$, each with $\operatorname{cdf} F(x)$. If these are rearranged in ascending order of magnitude and written as $X_{(1 ; n)} \leq X_{(2 ; n)} \leq \ldots$ $\leq X_{(n ; n)}$, then $X_{(r ; n)},(r=1,2, \ldots, n)$, is called the $r t h$ order statistic from a sample 
Review on Order Statistics and Record Values from $F^{\alpha}$ Distributions

of size $n$. Further $X_{(1 ; n)}, X_{(n ; n)}$ are called extreme order statistics, and $R=$ $X_{(n ; n)}-X_{(1 ; n)}$ is called the range. If $F_{(r ; n)}(x)$ and $f_{(r ; n)}(x),(r=1,2, \ldots, n)$, denote the cdf and probability density function (pdf) of the $r$ th order statistic $X_{(r ; n)}$ respectively, then these are defined as

$$
F_{(r ; n)}(x)=\sum_{j=r}^{n}\left(\begin{array}{l}
n \\
j
\end{array}\right)[F(x)]^{j}[1-F(x)]^{n-j}=I_{F(x)}(r, n-r+1),
$$

and

$$
f_{(r ; n)}(x)=\frac{1}{B(r, n-r+1)}[F(x)]^{r-1}[1-F(x)]^{n-r} f(x),
$$

where $I_{p}(a, b)=\frac{1}{B(a, b)} \int_{0}^{p} t^{a-1}(1-t)^{b-1} d t$ denotes the incomplete beta function. Many authors and researchers have studied the exact distributions of order statistics in samples of $n$ observations from various classes of distributions, among them, Sarhan and Greenberg (1962), Arnold et al. (1992), Ahsanullah and Nevzorov (2001), and David and Nagaraja (2003), are notable.

\subsection{Record Values: Definitions and Notations:}

Here we provide the distribution of record values. For detailed treatment of record values, the interested readers are referred to Ahsanullah (2004), and references therein.

Record Values: Suppose that $\left(X_{n}\right)_{n \geq 1}$ is a sequence of i.i.d. $r v^{\prime} s$ with cdf $F(x)$. Let $Y_{n}=\max (\min )\left\{X_{j} \mid 1 \leq j \leq n\right\}$ for $n \geq 1$. We say $X_{j}$ is an upper (lower) record value of $\left\{X_{n} \mid n \geq 1\right\}$, if $Y_{j}>(<) Y_{j-1}, j>1$. By definition $X_{1}$ is an upper as well as a lower record value.

Lower Record Values: The indices at which the lower record values occur are given by the record times $\{L(n), n \geq 1\}$, where $L(n)=\min$ $\left\{j \mid j>L(n-1), X_{j}<X_{L(n-1)}, n \leq 1\right\}$ and $L(1)=1$. We will denote $L(n)$ as the indices where the lower record values occur. The $n$th lower record value will be denoted by $X_{L(n)}$. If we define $F_{n}(x)$ as the c.d.f. of $X_{L(n)}$ for $n \geq 1$, then we have $F_{n}(x)=\int_{-\infty}^{x} \frac{(H(u))^{n-1}}{(n-1) !} d F(u), \quad-\infty<x<\infty$,

where $H(x)=-\ln F(x)$, and $\quad h(x)=-\frac{d}{d x} H(x)=f(x)(F(x))^{-1}$. The pdfof $\quad X_{L(n)}$, denoted by $f_{n}$, is

$$
f_{n}(x)=\frac{(H(x))^{n-1}}{\Gamma(n)} f(x), \quad-\infty<x<\infty .
$$


Upper Record Values: The indices at which the upper record values occur are given by the record times $\{U(n), n \geq 1\}$, where $U(n)=\min$ $\left\{j \mid j>U(n-1), X_{j}>X_{U(n-1)}, n>1\right\}$ and $U(1)=1$. Many properties of the upper record value sequence can be expressed in terms of the cumulative hazard rate function $R(x)=-\ln \bar{F}(x)$, where $\bar{F}(x)=1-F(x), 0<\bar{F}(x)<1$. If we define $F_{n}(x)$ as the cdf of $X_{U(n)}$ for $n \geq 1$, then we have

$$
F_{n}(x)=\int_{-\infty}^{x} \frac{\left(R(u) n^{n-1}\right.}{\Gamma(n)} d F(u), \quad-\infty<x<\infty,
$$

from which it is easy to see that

$F_{n}(x)=1-\bar{F}(x) \sum_{j=0}^{n-1} \frac{(R(x))^{j}}{\Gamma(j+1)}$

that is,

$$
\bar{F}_{n}(x)=\bar{F}(x) \sum_{j=0}^{n-1} \frac{(R(x))^{j}}{\Gamma(j+1)} .
$$

The pdf of $X_{U(n)}$, denoted by $f_{n}$ is

$$
f_{n}(x)=\frac{(R(x))^{n-1}}{\Gamma(n)} f(x), \quad-\infty<x<\infty .
$$

It is easy to see that $\bar{F}_{n}(x)-\bar{F}_{n-1}(x)=\bar{F}(x) \frac{f_{n}(x)}{f(x)}$. From here onward, for simplicity, the $n$th upper (lower) record value $X_{U(n)}$ (or, $X_{L(n)}$ ) will be denoted by $X(n)$.

\section{Record Values and Order Statistics from $F^{\alpha}$ Distributions}

\section{1. $F^{\alpha}$ Distributions: Definitions and Notations}

Definition: An absolutely continuous positive random variable $X$ is said to have $F^{\alpha}$ distribution if its cdf is given by $G(x)=F^{\alpha}(x)=[F(x)]^{\alpha}, \alpha>0, x>0$, which is the $\alpha$ th power of the base line distribution function $F(x)$. The distribution $G(x)$ is also called an exponentiated distribution of the given c.d.f. $F(x)$. Its pdf $g(x)$ is given by $g(x)=\alpha f(x) F^{\alpha-1}(x), \alpha>0, x>0$, where $f(x)=\frac{d F(x)}{d x}$ denotes the base line pdf of the random variable $X$.

Remarks: In literature, the $F^{\alpha}$ distribution is also defined as the proportional reversed hazard rate model (PRHRM) due to the fact that the reversed hazard 
Review on Order Statistics and Record Values from $F^{\alpha}$ Distributions

rate function (RHRF) of $G(x)$ is given by $\lambda_{G}^{*}(x)=\frac{d}{d x}(\ln (G(x)))=\frac{g(x)}{G(x)}$, where $g(x)$ is the pdf corresponding to $G(x)$.

Thus $\lambda_{G}^{*}(x)=\frac{\alpha(F(x))^{\alpha-1} f(x)}{(F(x))^{\alpha}}=\alpha \lambda_{F}^{*}(x)$, which implies that the RHRF of $G(x)$ is proportional to the RHRF of $F(x)$ with real constant of proportionality $\alpha$. When $\alpha$ is a positive integer, $F^{\alpha}$ is also defined as the Lehmann alternatives, that is, the model of non-parametric class of alternatives, see Lehmann (1953). The $k t h$ moment of the random variable $X$ having $F^{\alpha}$ distribution is given by

$$
\left.E\left[X^{k}\right)\right]=\int_{0}^{\infty} x^{k} g(x) d x=\int_{0}^{\infty} x^{k}\left[\alpha f(x) F^{\alpha-1}(x)\right] d x .
$$

Letting $F(x)=u$ in Eq. (3.1) and simplifying it, the expression for the $k$ th moment of $F^{\alpha}$ distribution is given by

$$
E\left[X^{k}\right]=\alpha \int_{0}^{1}\left[F^{-1}(u)\right]^{k} u^{\alpha-1} d u
$$

where $F^{-1}(u)$ represents the inverse of the base distribution $\operatorname{cdf} F(u)$ associated with $F^{\alpha}$ distribution. Thus, from (3.1), it is possible to determine the $k$ th moment of various distributions belonging to the family of $F^{\alpha}$ distribution provided the integral on the right side of the Eq. (3.2) exists and can be evaluated analytically in closed form or approximately.

3.2. Record Values and Order Statistics from $F^{\alpha}$ Distributions: Using Eq. (2.4), the $\operatorname{pdf} f_{n}$ and $\operatorname{cdf} F_{n}$ of the $n t h$ lower record value $X(n)$ from $F^{\alpha}$ distribution are, respectively, given by

$$
f_{n}(x)=\frac{\left[-\ln \left(F^{\alpha}(x)\right]^{n-1}\left[\alpha f(x) F^{\alpha-1}(x)\right]\right.}{\Gamma(n)}, \quad n=1,2,3, \ldots
$$

and

$$
F_{n}(x)=\frac{\Gamma\left(n,-\ln \left(F^{\alpha}(x)\right)\right)}{\Gamma(n)}, \quad n=1,2,3, \ldots
$$

where $x>0, \alpha>0$, and $\Gamma(c, z)=\int_{z}^{\infty} e^{-t} t^{c-1} d t, c>0$, denotes incomplete gamma function. The $k t h$ moment of the $n t h$ lower record value $X(n)$ with the pdf (3.3) is given by

$$
E\left[X^{k}(n)\right]=\int_{0}^{\infty} x^{k} \frac{\left[-\ln \left(F^{\alpha}(x)\right)\right]^{n-1}\left[\alpha f(x) F^{\alpha-1}(x)\right]}{\Gamma(n)} d x .
$$

Letting $-\ln \left(F^{\alpha}(x)\right)=u$ in Eq. (3.5) and simplifying it, the expression for the kth moment of the $n t h$ lower record value $X(n)$ is easily obtained as

$$
E\left[X^{k}(n)\right]=\frac{1}{\Gamma(n)} \int_{0}^{\infty}\left[F^{-1}(\exp (-u / \alpha))\right]^{k} u^{n-1} \exp (-u) d u
$$


where $F^{-1}(z)$ represents the inverse of the base distribution $\operatorname{cdf} F(z)$ associated with $F^{\alpha}$ distribution. Thus, from (3.6), it is possible to determine the $k t h$ moment of the $n t h$ lower record value $X(n)$ from various distributions belonging to the family of $F^{\alpha}$ distribution provided the integral on the right side of the Eq. (3.6) exists and can be evaluated analytically in closed form or approximately.

Using Eq. (2.1) and Eq. (2.2), the pdf $f_{n}, \operatorname{cdf} F_{n}$ and $k t h$ moment of the $r$ th order statistic $X_{(r ; n)}, 1 \leq r \leq n$, from $F^{\alpha}$ distribution, can similarly be expressed as

$$
\begin{aligned}
& F_{(r ; n)}(x)=\sum_{j=r}^{n}\left(\begin{array}{l}
n \\
j
\end{array}\right)[F(x)]^{\alpha j}\left[1-F^{\alpha}(x)\right]^{n-j}, \\
& f_{(r ; n)}(x)=\frac{\alpha}{B(r, n-r+1)} f(x)[F(x)]^{\alpha r-1}\left[1-F^{\alpha}(x)\right]^{n-r},
\end{aligned}
$$

and

$$
E\left[X_{(r ; n)}^{k}\right]=\frac{1}{B(r, n-r+1)} \int_{0}^{1} u^{r-1}(1-u)^{n-r}\left[F^{-1}\left(u^{1 / \alpha}\right)\right]^{k} d u,
$$

where $F^{\alpha}(x)=u$.

In what follows, we will discuss some distributional properties of record values and order statistics from various distributions belonging to the family of $F^{\alpha}$ distribution.

\subsection{Generalized Exponential (GE) or Exponentiated Exponential (EE) Distribution}

A random variable $X$ is said to have the EE distribution if its cdf is given by

$$
G(x)=(1-\exp (-\lambda x))^{\alpha},
$$

for $x>0, \lambda>0$ and $\alpha>0$, which is the $\alpha$ th power of the cdf of the standard exponential distribution. The corresponding pdf of the EE distribution (3.7) is given by

$$
g(x)=\alpha \lambda \exp (-\lambda x)[1-\exp (-\lambda x)]^{\alpha-1}
$$

where $\alpha$ and $\lambda$ are the shape and scale parameters, respectively. It is easy to see that the mean and variance of the random variable $X$ with pdf (3.7) are, respectively, given by

$$
E(X)=\psi(\alpha+1)-\psi(1),
$$

and

$$
V(X)=\psi^{\prime}(1)-\psi^{\prime}(\alpha+1),
$$

where $\psi($.$) and its derivative \psi^{\prime}($.$) denote psi (or digamma) and polygamma$ functions, respectively, see Gupta and Kundu (1999, 2000, 2001), and 
Nadarajah(2011). The possible shapes of the pdf (3.7), when $\lambda=1$, are provided for some selected values of the parameter $\alpha$, that is, for $\alpha=0.5,2,5,10,50$, in

Fig. 3.1 below.
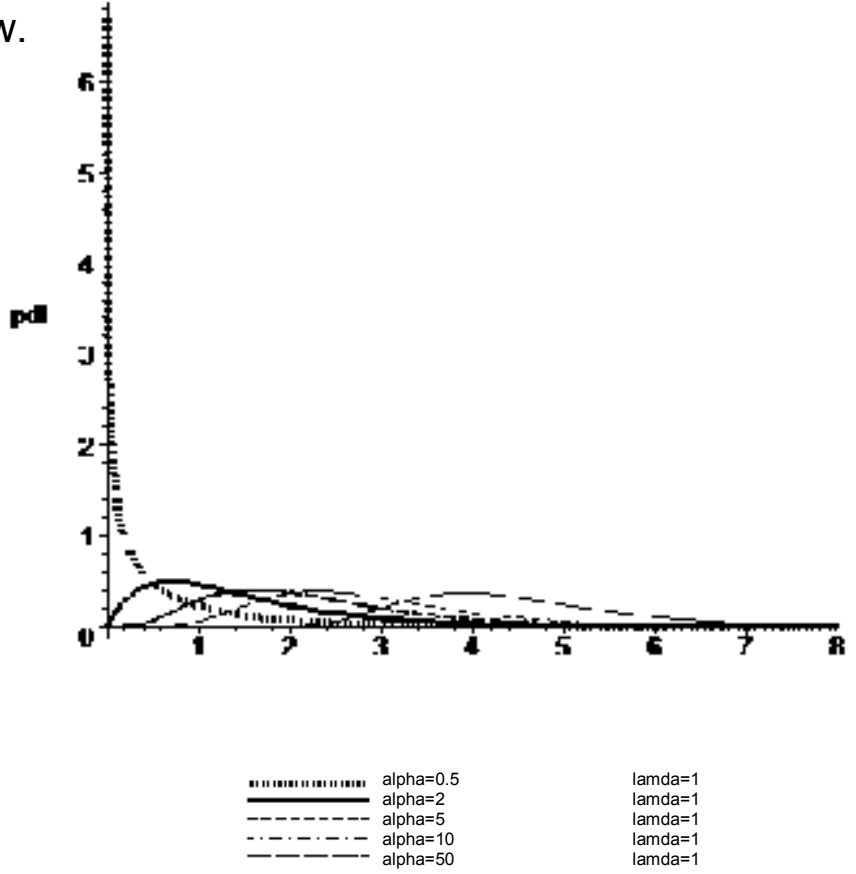

Figure 3.1: PDF Plots of EE Distribution for $\alpha=0.5,2,5,10,50$ when $\lambda=1$

The effects of the parameters can easily be seen from the graph. For example, it is clear from the plotted Fig. 3.1, for selected values of the parameter $\alpha$, that the pdf of the EE distribution can take different shapes. It is unimodal for $\alpha>1$ and reversed ' $\mathrm{J}$ ' shaped for $\alpha \leq 1$. Further, the density function of the EE distribution is log-convex if $\alpha \leq 1$ and log-concave if $\alpha \geq 1$. Also, it has an increasing or decreasing hazard function if $\alpha>1$ or $\alpha<1$, respectively, and for $\alpha=1$, the hazard function is constant.

Order Statistics: For details on order statistics from GE (or EE) distribution, the interested readers are referred to Raqab and Ahsanullah (2001), where different order statistics of a three parameter GE distribution, moment generating functions and product moments, and the best linear unbiased estimators of the location and scale parameters have been considered. Also, see Gupta and Kundu $(1999,2007)$, Sultan (2007), and Nadarajah (2011). Let $X_{1}, X_{2}, \ldots, X_{n}$ be a random sample from the GE distribution with cdf and pdf as (3.7) and (3.8), respectively, and let $X_{(1 ; n)} \leq X_{(2 ; n)} \leq \ldots \leq X_{(n ; n)}$ denote the order statistics obtained from this sample. One can easily obtain the pdf $f_{(r ; n)}(x)$ and $\operatorname{cdf} F_{(r ; n)}(x)$ of the $r$ th order statistic $X_{(r ; n)}, 1 \leq r \leq n$, from the EE distribution byusing Eqs. (3.7) and (3.8) in (2.2) and (2.1), respectively. Thus, if the random variable $X$ has the pdf (3.8) with $\lambda=1$, then, following the notations of Raqaband 
Ahsanullah (2001), the pdf of the $r$ th order statistic $X_{(r ; n)}, 1 \leq r \leq n$, for $x>0$, is easily obtained as

$$
f_{(r ; n)}(x)=\sum_{j=0}^{n-r} d_{j}(n, r) f\left(x ; \alpha_{r+j}\right),
$$

where $\alpha_{j}=j \alpha, d_{j}(n, r)=\frac{(-1)^{j} n\left(\begin{array}{c}n-1 \\ r-1\end{array}\right)\left(\begin{array}{c}n+r \\ j\end{array}\right)}{r+j}$, and $f\left(x ; \alpha_{r+j}\right)$ denotes the EE distribution with the shape parameter $\alpha(r+j)$ and scale parameter 1 . Note that the coefficients $d_{j}(n, r), \quad(j=1,2, \ldots, n-r)$, are not dependent on $\alpha$. The moment generating function, $M_{X_{(r ; n)}}(t)$, of $X_{(r ; n)}$, for $|t|<1$, is easily given by $M_{X_{(r ; n)}}(t)=E\left(\exp \left(t X_{(r ; n)}\right)\right)=\int_{0}^{\infty} \exp (t x) f_{(r ; n)}(x) d x=\frac{\alpha}{B(r, n-r+1)} \sum_{j=0}^{n-r}(-1)^{j}$ $\left(\begin{array}{c}n-r \\ j\end{array}\right) \frac{\Gamma(\alpha(r+j)) \Gamma(1-t)}{\Gamma(\alpha(r+j)-t+1)},|t|<1$.

Differentiating $M_{X_{(r ; n)}}(t)$ twice with respect to $t$ and evaluating at $t=0$, it is easy to see that the mean (first moment) and the second moment of $X_{(r ; n)},(r=1,2, \ldots, n)$, are, respectively, given by

$$
\begin{aligned}
& \alpha_{r ; n}=E\left(X_{(r ; n)}\right)=\frac{1}{B(r, n-r+1)} \sum_{j=0}^{n-r}(-1)^{j} \frac{\left(\begin{array}{c}
n-r \\
j
\end{array}\right)}{(r+j)}[\psi(\alpha(r+j)+1)+\gamma], \quad \text { and } \\
& \alpha_{r ; n}^{(2)}=E\left(X_{(r ; n)}^{2}\right)=\frac{1}{B(r, n-r+1)} \sum_{j=0}^{n-r}(-1)^{j} \\
& \frac{\left(\begin{array}{c}
n-r \\
j
\end{array}\right)}{(r+j)}\left[\{\psi(\alpha(r+j)+1)+\gamma\}^{2}+\psi^{\prime}(1)-\psi^{\prime}(\alpha(r+j)+1)\right],
\end{aligned}
$$

where $\psi(z)=\frac{d(\log \Gamma(z))}{d z}$ denotes digamma function, $\gamma=-\psi(1)=0.577215 \ldots$ is the Euler's constant, and $\psi^{\prime}(1)=\zeta(2)=\pi^{2} / 6$, where $\psi^{\prime}(z)$ and $\zeta(z)$ are called trigamma and Riemann zeta functions, respectively, (see Abramowitz and Stegun, 1970, pages 260 and 807).

Record Values: For details on record values from GE (or EE) distribution, the interested readers are referred to Raqab (2002) where a three parameter (including the location) GE distribution and the best linear unbiased estimators of the location and scale parameters using the moments of the record statistics have been considered. See also Asgharzadeh and Valiollahi (2010), and Asgharzadeh and Fallah (2011). The pdf $f_{n}$ and $\operatorname{cdf} F_{n}$ of the $n t h$ lower record value $X(n)$ from EE distribution can easily be obtained byusing Eqs. (3.7) and (3.8) in (3.3) and (3.4), respectively. If the random variable $X$ has the pdf (3.8) with $\lambda=1$, then, following Raqab (2002), the moment generating function, $M(t)$, of the $n t h$ lower record value $X(n)$ is easily given by

where

$$
M_{X(n)}(t)=E(\exp (t) X(n))=\int_{0}^{\infty} \exp (t x) f_{n}(x) d x=\alpha^{n} \sum_{j=0}^{\infty} \frac{(t)_{j}}{j !} \frac{1}{(\alpha+j)^{n}}, \quad t \neq 0,
$$

$$
(t)_{j}=t(t+1) \ldots(t+j-1), j=1,2,3, \ldots, \text { and }(t)_{0}=1 .
$$

Thus differentiating $M_{X(n)}(t)$ and evaluating at $t=0$, the mean and the second moment of the $n$th lower record value $X(n)$ are easily obtained as

$$
E(X(n))=\sum_{j=1}^{\infty} \frac{\alpha^{n}}{j(\alpha+j)^{n}}, \quad n \geq 1,
$$


and

$$
E\left(X^{2}(n)\right)=\alpha^{n} \sum_{j=1}^{\infty} \sum_{k=1}^{\infty} \frac{\alpha^{n}}{j k(j+k+\alpha)^{n}} .
$$

For exact derivation of single and product moments of record statistics and recurrence relations between these moments, and uses of these in the evaluations of the means, variances and covariances for different values of the shape parameter $\alpha$, one is referred to Raqab (2002).

\subsection{ExponentiatedWeibull (EW) distribution:}

The EW distribution was introduced by Mudholkar and Srivastava (1993) as an extension of the Weibull family. Various properties and applications of the EW distribution have also been studied by many authors, among them Mudholkar et al. (1995), Mudholkar and Hutson (1996), Nassar and Eissa (2003), Pal et al. (2006), Nadarajah and Gupta (2005), and Nadarajah and Kotz (2006) are notable. A random variable $X$ is said to have the exponentiatedWeibull distribution if its cdf and pdf are respectively given by

and

$$
G(x)=\left[1-\exp \left(-(\lambda x)^{\delta}\right)\right]^{\alpha}
$$

$$
g(x)=\alpha \delta \lambda^{\delta} x^{\delta-1} \exp (-\lambda x)^{\delta}\left[1-\exp \left(-(\lambda x)^{\delta}\right)\right]^{\alpha-1},
$$

for $x>0, \lambda>0, \delta>0$ and $\alpha>0$. The kth moment of the EW random variable $X$ with distribution function (3.9) is given by

$$
E\left[X^{k}\right]=\alpha \lambda^{-k} \Gamma\left(\frac{k}{\delta}+1\right) \sum_{j=0}^{\infty} \frac{(1-\alpha)_{j}}{j !(j+1)^{(k-\delta) / \delta}},
$$

for any $n>-\delta$, where $(1-\alpha)_{j}=(-1)^{j} \frac{\Gamma(\alpha)}{\Gamma(\alpha-j)}$, from which the mean and variance of the random variable $X$ can easily be obtained. The possible shapes of the pdf (3.10) are provided for some selected values of the parameters in Fig. 3.2 for $\lambda=1, \delta=2$, and $\alpha=0.5,2,5,10,50$, and in Fig. 3.3 for $\lambda=1, \delta=5$ and $\alpha=0.5,2,5,10,50$, respectively.

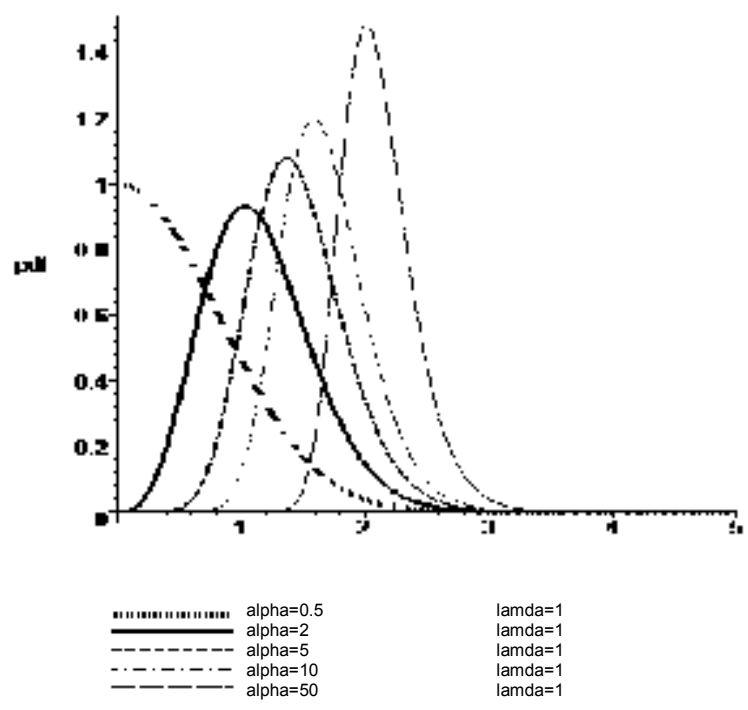

Figure 3.2: PDF Plots of EW Distribution for $\alpha=0.5,2,5,10,50$ when $\lambda=1$ and $\delta=2$ 


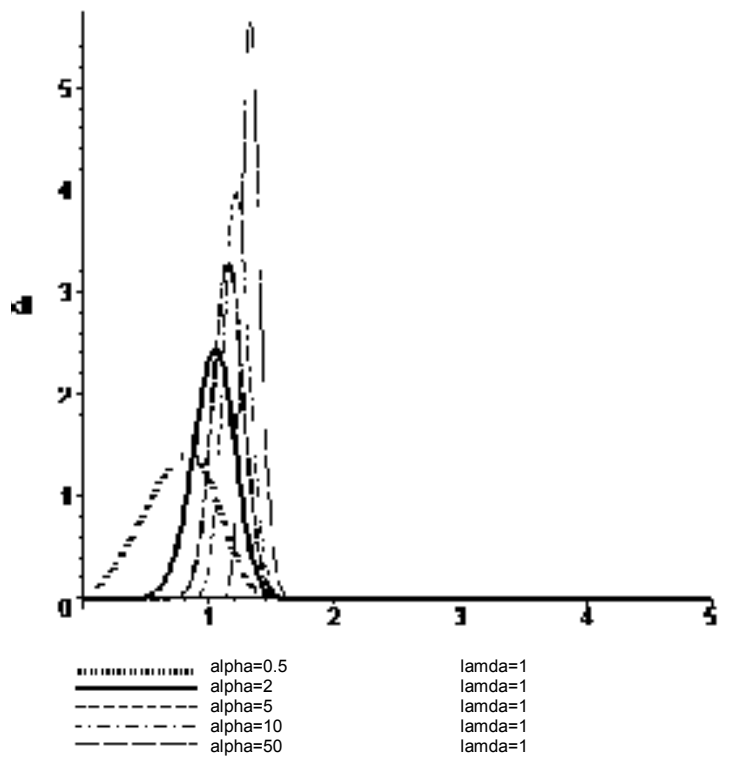

Figure 3.3: PDF Plots of EW Distribution for $\alpha=0.5,2,5,10,50$ when $\lambda=1$ and $\delta=5$

The effects of the parameters can easily be seen from the graph. It is clear from both Fig. 3.2 and Fig. 3.3 that the pdf of EW distribution is unimodal and, for fixed $\lambda$ and $\delta$, it becomes more and more symmetric as $\alpha$ increases.It appears from the literature that not much has been done on the order statistics and record values from EW distribution. However, we derive the pdf $f_{n}$ and cdf $F_{n}$ of the $n t h$ lower record value $X(n)$ from EW distribution, which, byusing Eqs. (3.9) and (3.10) in (3.3) and (3.4), respectively, are easily given as

$$
f_{n}(x)=\frac{\left[-\ln \left(\left[1-\exp \left(-(\lambda x)^{\delta}\right)\right]^{\alpha}\right)\right]^{n-1}\left[\alpha \delta \lambda^{\delta} x^{\delta-1} \exp (-\lambda x)^{\delta}\left[1-\exp \left(-(\lambda x)^{\delta}\right)\right]^{\alpha-1}\right]}{\Gamma(n)},
$$

and

$$
F_{n}(x)=\frac{\Gamma\left(n,-\ln \left(\left[1-\exp \left(-(\lambda x)^{\delta}\right)\right]^{\alpha}\right)\right)}{\Gamma(n)},
$$

where $n>0$ (an integer), $x>0, \lambda>0, \delta>0, \alpha>0$, and $\Gamma(c, z)=\int_{z}^{\infty} e^{-t} t^{c-1} d t$, $c>0$, denotes incomplete gamma function. The $k t h$ moment of the $n t h$ lower record value $X(n)$ from $\mathrm{EW}$ distribution is given by

$$
E\left[X^{k}(n)\right]=\int_{0}^{\infty} x^{k} \frac{\left[-\ln \left(\left[1-\exp \left(-(\lambda x)^{\delta}\right)\right]^{\alpha}\right)\right]^{n-1}\left[\alpha \delta \lambda^{\delta} x^{\delta-1} \exp (-\lambda x)^{\delta}\left[1-\exp \left(-(\lambda x)^{\delta}\right)\right]^{\alpha-1}\right]}{\Gamma(n)} d x .
$$

Letting $-\ln \left(\left[1-\exp \left(-(\lambda x)^{\delta}\right)\right]^{\alpha}\right)=u$ in the above Eq. and simplifying it, the expression for the $k t h$ moment of the $n t h$ lower record value $X(n)$ is obtained as

$$
E\left[X^{k}(n)\right]=\frac{1}{\lambda^{k} \Gamma(n)} \int_{0}^{\infty}[-\ln (1-\exp (-u / \alpha))]^{k / \delta} u^{n-1} \exp (-u) d u,
$$

which, in view of the fact that $-\ln (1-\exp (-u / \alpha))=\sum_{j=1}^{\infty} \frac{\exp (-j u / \alpha)}{j}$, reduces to

$$
E\left[X^{k}(n)\right]=\frac{1}{\lambda^{k} \Gamma(n)} \int_{0}^{\infty}\left[\sum_{j=1}^{\infty} \frac{\exp (-j u / \alpha)}{j}\right]^{k / \delta} u^{n-1} \exp (-u) d u .
$$


Review on Order Statistics and Record Values from $F^{\alpha}$ Distributions

It is evident from the above expression that the analytical derivations of the exact or tractable closed form expressions for the moments of record statistics from EW distribution seem to be complicated, and therefore need further investigations. However, for $\delta=1$, since the $\mathrm{EW}$ distribution reduces to $\mathrm{EE}$ distribution, one is referred to Raqab (2002) for exact derivation of the moments of record statistics. Similarly, one can obtain the pdf $f_{(r ; n)}(x)$ and $\operatorname{cdf} F_{(r ; n)}(x)$ of the $r$ th order statistic $X_{(r ; n)}, 1 \leq r \leq n$, from EW distribution byusing Eqs. (3.9) and (3.10) in (2.1) and (2.2), respectively. Note that the analytical derivations of the exact or tractable closed form expressions for the moments of order statistics from EW distribution are complicated, and therefore also need further investigations.

3.5. Power Function Distribution: A random variable $X$ is said to have the power function distribution if its cdf and pdf are, respectively, given by

$$
G(x)=\left(\frac{x}{\lambda}\right)^{\alpha},
$$

and

$$
g(x)=\frac{\alpha}{\lambda}\left(\frac{x}{\lambda}\right)^{\alpha-1},
$$

where $\lambda>0, \alpha>0$, and $0<x<\lambda$. For statistical properties of power function distribution, see, for example, Johnson et al. (1994). For a discussion on order statistics from power function distribution, the interested readers are referred to Ahsanullah and Nevzorov (2001). See also Malik (1967). For record values from power function distribution, one is referred to Ahsanullah (2004), where distributional properties of upper record values from a three parameter power function distribution, recurrence relation between moments, estimation of the parameters and prediction of record values have been considered. The pdf $f_{n}$ and $\operatorname{cdf} F_{n}$ of the $n t h$ lower record value $X(n)$ from power function distribution can easily obtained byusing Eqs. (3.11) and (3.12) in (3.3) and (3.4), respectively, as $\quad f_{n}(x)=\frac{\left[-\ln (x / \lambda)^{\alpha}\right]^{k-1}\left[(\alpha / \lambda)(x / \lambda)^{\alpha-1}\right]}{\Gamma(n)}$,

and $F_{n}(x)=\frac{\Gamma\left(n,-\ln (x / \lambda)^{\alpha}\right)}{\Gamma(n)}$,

where $n>0$ (an integer), $0<x<\lambda, \lambda>0$ and $\alpha>0$, and $\Gamma(c, z)=\int_{z}^{\infty} e^{-t} t^{c-1} d t$, $c>0$, denotes incomplete gamma function. The $k t h$ moment of the $n t h$ lower record value $X(n)$ from power function distribution is easily given by

$$
E\left[X^{k}(n)\right]=\int_{0}^{\lambda} x^{k} \frac{\left[-\ln (x / \lambda)^{\alpha}\right]^{n-1}\left[(\alpha / \lambda)(x / \lambda)^{\alpha-1}\right]}{\Gamma(n)} d x .
$$

Letting $-\ln (x / \lambda)^{\alpha}=u$ in the above Eq. and simplifying it, the expression for the $k t h$ moment of the $n t h$ lower record value $X(n)$ is easily obtained as

$$
E\left[X^{k}(n)\right]=\frac{\lambda^{k} \alpha^{n}}{(\alpha+k)^{n}},
$$


from which the single moment (that is, the mean), the second single moment and variance of the $n t h$ lower record value $X(n)$ from power function distribution are easily given as

and

$$
\begin{aligned}
& E[X(n)]=\frac{\lambda \alpha^{n}}{(\alpha+1)^{n},} \\
& E\left[X^{2}(n)\right]=\frac{\lambda^{2} \alpha^{n}}{(\alpha+2)^{n}},
\end{aligned}
$$

$$
\operatorname{Var}[X(n)]=\lambda^{2} \alpha^{n}\left\lfloor\frac{1}{(\alpha+2)^{n}}-\frac{\alpha^{n}}{(\alpha+1)^{2 n}}\right\rfloor
$$

Similarly, one can obtain the pdf $f_{(r ; n)}(x)$ and $\operatorname{cdf} F_{(r ; n)}(x)$ of the $r$ th order statistic $X_{(r ; n)}, \quad 1 \leq r \leq n$, from power function distribution byusing Eqs. (3.11) and (3.12) in (2.2) and (2.1), respectively. We can also derive expressions for the moments of order statistics from power function distribution (3.11). Thus, if the random variable $X$ has the pdf (3.11), the $k t h$ moment of the $r$ th order statistic $X_{(r ; n)}$ from a sample of size $n$ is given by

$$
E\left(X_{(r ; n)}^{k}\right)=\frac{\Gamma(n+1) \Gamma\left(\frac{k}{\alpha}+r\right) \lambda^{k}}{\Gamma(r) \Gamma\left(n+\frac{k}{\alpha}+1\right)}, r=1,2,3, \ldots, n,
$$

where $\Gamma(z)$ denotes gamma function. Also we have the following recurrence relation between moments of the order statistics $X_{(r ; n)}$ and $X_{(r-1 ; n)}$ :

$$
E\left(X_{(r ; n)}^{k}\right)=\frac{\left(r+\frac{k}{\alpha}-1\right) E\left(X_{(r-1 ; n)}^{k}\right)}{(r-1)}, r=1,2,3, \ldots, n, k>1 .
$$

Using the above Eqs., the single moment (that is, the mean), the second single moment and variance of the $r t h$ order statistic $X_{(r ; n)}, 1 \leq r \leq n$, from power function distribution, can easily be derived.

3.6. Pareto Type II (or Exponentiated Pareto or Lomax) Distribution: The Pareto type II distribution is defined by the cdf

$$
G(x)=\left[1-\left(\frac{1}{1+\lambda x^{\delta}}\right)\right]^{\alpha},
$$

for $x>0, \delta>0, \lambda>0$ and $\alpha>0$, and was introduced by Pickands (1975) to study extreme events. See also Ahsanullah (2004). Another variant of the Pareto type II or the exponentiated Paretodistribution was introduced by Gupta et al. (1998) as a lifetime model with the cdf and pdf expressed, respectively, as

$$
G(x)=\left[1-\left(\frac{1}{1+x}\right)^{\beta}\right]^{\alpha}
$$

and

$$
g(x)=\alpha \beta\left[1-\left(\frac{1}{1+x}\right)^{\beta}\right]^{\alpha-1}\left(\frac{1}{1+x}\right)^{-(\beta+1)},
$$

for $x>0, \beta>0$ and $\alpha>0$, where $\alpha$ and $\beta$ denote the shape parameters of the exponentiated Paretodistribution given by (3.14). When $\alpha=1$, the above distribution (3.14) corresponds to the standard Pareto distribution of the second kind, see Johnson et al. (1994). See also Shawky and Abu-Zinadah (2008, 2009), Ali and Woo (2010), and Afify (2010) for other distributional properties and applications of the Pareto type II distribution. The pdf $f_{n}$ and $\operatorname{cdf} F_{n}$ of the $n t h$ 
Review on Order Statistics and Record Values from $F^{\alpha}$ Distributions

lower record value $X(n)$ from $E P(\alpha, \beta)$ can easily obtained byusing the Eqs. (3.14) and (3.15) in (3.3) and (3.4), respectively. Similarly, one can obtain the pdf $f_{(r ; n)}(x)$ and $\operatorname{cdf} F_{(r ; n)}(x)$ of the $r$ th order statistic $X_{(r ; n)}, 1 \leq r \leq n$, from $E P(\alpha, \beta)$ byusing the Eqs. (3.14) and (3.15) in (2.2) and (2.1), respectively. It appears from the literature that not much attention has been paid on the order statistics and record values from $\operatorname{EP}(\alpha, \beta)$. Recently, Shawky and Abu-Zinadah (2009) studied the estimation of the parameters of the order statistics from $E P(\alpha, \beta)$, and compared the performances of different estimation procedures through numerical simulations.

Also, Ali and Woo (2010) studied some distributional properties and estimation problems of the first order statistics for a special case of the exponentiated Pareto distribution $\operatorname{EP}(\alpha, \beta)$. For the derivation of exact form of the pdf, moments of single, double, triple and quadruple of lower record values and recurrence relations between single, double, triple and quadruple moments of lower record values from $E P(\alpha, \beta)$, the interested readers are referred to Shawky and AbuZinadah (2008).

\subsection{Burr Type $X$ or Generalized Rayleigh (GR) Distribution}

The two-parameter Burr type $\mathrm{X}$ (also called the generalized Rayleigh distribution, denoted by $G R(\alpha, \lambda)$, where $\alpha$ and $\lambda$ denote the shape and scale parameters respectively) was introduced by Surles and Padgett (2001) to model strength and lifetime data. Its cdf and pdf are, respectively, expressed as

$G(x)=\left(1-\exp \left(-(\lambda x)^{2}\right)\right)^{\alpha}$,

and

$g(x)=2 \alpha \lambda^{2} x \exp (-\lambda x)^{2}\left[1-\exp \left(-(\lambda x)^{2}\right)\right]^{\alpha-1}$,

for $x>0, \lambda>0$ and $\alpha>0$. Note that the $G R(\alpha, \lambda)$ distribution is a special case of the EW distribution, as defined above. It is observed in Raqab and Kundu (2006) that for $\alpha \leq 0.5$, the pdf (3.17) of $G R(\alpha, \lambda)$ distribution is a decreasing function. It is right-skewed and unimodalfor $\alpha>0.5$. Further, the hazard function of $G R(\alpha, \lambda)$ distribution is bathtub type for $\alpha \leq 0.5$ and is increasing for $\alpha>0.5$. It appears from the literature that not much has been paid on the record values from generalized Rayleigh distribution. Byusing Eqs. (3.16) and (3.17) in (3.3) and (3.4), respectively, the pdf $f_{n}$ and $\operatorname{cdf} F_{n}$ of the $n t h$ lower record value $X(n)$ from $G R(\alpha, \lambda)$ distribution can easily be obtained as

$$
f_{n}(x)=\frac{\left[-\ln \left(\left[1-\exp \left(-(\lambda x)^{2}\right)\right]^{\alpha}\right)\right]^{n-1}\left[2 \alpha \lambda^{2} x \exp (-\lambda x)^{2}\left[1-\exp \left(-(\lambda x)^{2}\right)\right]^{\alpha-1}\right]}{\Gamma(n)},
$$

and

$$
F_{n}(x)=\frac{\Gamma\left(n,-\ln \left(\left[1-\exp \left(-(\lambda x)^{2}\right)^{\alpha}\right)\right)\right.}{\Gamma(n)},
$$


where $n>0$ (an integer), $x>0, \lambda>0, \alpha>0$, and $\Gamma(c, z)=\int_{z}^{\infty} e^{-t} t^{c-1} d t, c>0$, denotes incomplete gamma function. The $k$ th moment of the $n t h$ lower record value $X(n)$ from $G R(\alpha, \lambda)$ distribution is given by

$$
E\left[X^{k}(n)\right]=\int_{0}^{\infty} x^{k} \frac{\left[-\ln \left(\left[1-\exp \left(-(\lambda x)^{2}\right)\right]^{\alpha}\right)\right]^{n-1}\left[2 \alpha \lambda^{2} x \exp (-\lambda x)^{2}\left[1-\exp \left(-(\lambda x)^{2}\right)\right]^{\alpha-1}\right]}{\Gamma(n)} d x .
$$

Letting $-\ln \left(\left[1-\exp \left(-(\lambda x)^{2}\right)\right]^{\alpha}\right)=u$ in the above Eq. and simplifying it, the expression for the $k$ th moment of the $n t h$ lower record value $X(n)$ is obtained as

$$
E\left[X^{k}(n)\right]=\frac{1}{\lambda^{k} \Gamma(n)} \int_{0}^{\infty}[-\ln (1-\exp (-u / \alpha))]^{k / 2} u^{n-1} \exp (-u) d u
$$

which, in view of the fact that $-\ln (1-\exp (-u / \alpha))=\sum_{j=1}^{\infty} \frac{\exp (-j u / \alpha)}{j}$, reduces to

$$
E\left[X^{k}(n)\right]=\frac{1}{\lambda^{k} \Gamma(n)} \int_{0}^{\infty}\left[\sum_{j=1}^{\infty} \frac{\exp (-j u / \alpha)}{j}\right]^{k / 2} u^{n-1} \exp (-u) d u .
$$

It is evident from the above expression that the analytical derivation of the exact or tractable closed form expressions for the moments of record statistics from $G R(\alpha, \lambda)$ distribution is complicated, and, therefore, needs further investigations. For a discussion on order statistics from $G R(\alpha, \lambda)$ distribution, the interested readers are referred to Raqab (1998), and Kundu and Raqab (2005). By using Eqs. (3.16) and (3.17) in (2.1) and (2.2) respectively, the cdf $F_{(r ; n)}(x)$ and pdf $f_{(r ; n)}(x)$ of the $r$ th order statistic $X_{(r ; n)}, 1 \leq r \leq n$ from $G R(\alpha, \lambda)$ distribution are given by

$$
F_{(r ; n)}(x)=\sum_{j=r}^{n}\left(\begin{array}{l}
n \\
j
\end{array}\right)\left[\frac{\Gamma\left(n,-\ln \left(\left[1-\exp \left(-(\lambda x)^{2}\right)\right]^{\alpha}\right)\right)}{\Gamma(n)}\right]^{j}\left[1-\frac{\Gamma\left(n,-\ln \left(\left[1-\exp \left(-(\lambda x)^{2}\right)\right]^{\alpha}\right)\right)}{\Gamma(n)}\right]^{n-j},
$$

and

$$
\begin{aligned}
& f_{(r ; n)}(x)=\frac{1}{B(r, n-r+1)}\left[\frac{\Gamma\left(n,-\ln \left(\left[1-\exp \left(-(\lambda x)^{2}\right)\right]^{\alpha}\right)\right)}{\Gamma(n)}\right]^{r-1}\left[1-\frac{\Gamma\left(n,-\ln \left(\left[1-\exp \left(-(\lambda x)^{2}\right)\right]^{\alpha}\right)\right)}{\Gamma(n)}\right]^{n-r} \times \\
& \times \frac{\left[-\ln \left(\left[1-\exp \left(-(\lambda x)^{2}\right)\right]^{\alpha}\right)\right]^{n-1}\left[2 \alpha \lambda^{2} x \exp (-\lambda x)^{2}\left[1-\exp \left(-(\lambda x)^{2}\right)\right]^{\alpha-1}\right]}{\Gamma(n)}
\end{aligned}
$$

For a discussion on order statistics from $G R(\alpha, 1)$ distribution, such as the exact form of the pdf, moments, measures of skewness and kurtosis, percentage points and estimation of parameters, one is referred to Raqab (1998). 


\section{Record Values and Order Statistics from Other Members of $F^{\alpha}$ Family of Distributions}

Besides the above stated $F^{\alpha}$ distributions in Section 3, several other members of $F^{\alpha}$ family of distributions have recently been developed by various authors. For the sake of completeness, we briefly describe some of these members of the $F^{\alpha}$ family of distributions.

\subsection{Generalized Logistic (GL) Distribution:}

The two-parameter generalized logistic distribution, denoted by $G L(\alpha, \lambda)$, where $\alpha$ and $\lambda$ denote the shape and scale parameters, respectively, was introduced by Balakrishnan and Leung (1988) as one of the three generalized forms of the standard logistic distribution. Its cdf and pdf are respectively expressed as

$$
G(x)=\frac{1}{\left(1+e^{-\lambda x}\right)^{\alpha}},
$$

and

$$
g(x)=\frac{\alpha \lambda e^{-\lambda x}}{\left(1+e^{-\lambda x}\right)^{\alpha+1}},
$$

for $-\infty<x<\infty, \lambda>0$ and $\alpha>0$. The $G L(\alpha, \lambda)$ distribution was originally proposed as a generalization of the logistic distribution by Ahuja and Nash (1967). See also Johnson et al. (1994). As observed by Alkasasbeh and Raqab (2009), the logistic distribution has been used for growth models and is used in a certain type of regression known as the logistic regression. It also has applications in modeling life data. This model is also used in survival analysis as a parametric model for events whose rate increases initially and decreases later, for example mortality from cancer following diagnosis or treatment. It has also been used in hydrology to model stream flow and precipitation, and in economics as a simple model of the distribution of wealth or income. It is observed by Balakrishnan and Leung (1988) that $G L(\alpha, \lambda)$ is skewed and its kurtosis coefficient is greater than that of the logistic distribution. For $\alpha=1$, the GL distribution becomes the standard logistic and is symmetric. Further, Alkasasbeh and Raqab (2009) observed that the pdf (4.2) of $G L(\alpha, \lambda)$ distribution is increasing for $x<\left(\lambda^{-1} \ln \alpha\right)$ and is decreasing for $x>\left(\lambda^{-1} \ln \alpha\right)$. Therefore it is a unimodal distribution with mode at $\ln \alpha$. The density function of $G L(\alpha, \lambda)$ is logconcave for all values of $\alpha$. For $\alpha>1$, it is positively skewed and for $0<\alpha<1$, it is negatively skewed. For this, the proportionality constant $\alpha$ can be represented as the skewness parameter. The hazard rate of GL distribution can be either bathtub type or an increasing function, depending on the shape parameter $\alpha$. For $\alpha<1$, the hazard rate of $G L(\alpha, \lambda)$ is bathtub type and for $\alpha \geq 1$ , it is an increasing function. For other statistical properties, different estimation procedures and applications of the $G L(\alpha, \lambda)$ distribution, the interested readers are referred to Balakrishnan (1990), Chen and Balakrishnan(1995), and Asgharzadeh (2006). 


\subsection{The Exponentiated Generalized Inverse Gaussian (EGIG) Distribution}

Recently, Lemonte and Cordeiro (2011) have introduced the exponentiated generalized inverse Gaussian (EGIG) distribution. Additionally, as a particular case of EGIG, they also introduced the exponentiated gamma (EGamma) distribution, which generalizes the exponentiated standard gamma (ESGamma) distribution proposed by Nadarajah and Kotz (2006). See also Shawky and Bakoban $(2008,2009)$ and Shadrokh and Pazira (2011) who studied statistical properties and applications of the EGamma distribution.

\subsection{Other Members}

Nadarajah and Kotz (2006) also proposed four more members of $F^{\alpha}$ family of distributions to extend the standard gamma, standard Weibull, standard Gumbel and standard Fréchet distributions. See also Persson and Ryden (2010) for exponentiatedGumbel distribution and its application in estimation of return levels of significant wave height. Cho et al. (2009) studied the properties of the exponentiated extreme value distribution. Silva et al. (2010) introduced the exponentiated exponential geometric distribution (also known as the generalized exponential geometric (GEG) distribution). Recently, the exponentiated inverse Weibull distribution has been introduced by Gusmão et al. (2011).

\subsection{Remarks}

It appears from the literature that not much attention has been paid to the analysis of order statistics and record values from the above-mentioned recently introduced members of $F^{\alpha}$ family of distributions, and, therefore, needs further and special investigations.

\section{Concluding Remarks}

It is evident from the above examples of $F^{\alpha}$ distributions that adding a parameter, say, $\alpha>0$, to a $\operatorname{cdf} F$ by exponentiation produces a $\operatorname{cdf} F^{\alpha}$ which is richer and more flexible to modeling data. It has been observed that $F^{\alpha}$ is flexible enough to accommodate both monotone as well as non-monotone hazard rates. For example, if $F$ is exponential such that $F(x)=1-\exp (-\lambda x)$, then its $\operatorname{pdf} f(x)=\lambda \exp (-\lambda x)$ is monotone decreasing on the positive half of the real line. However, $G(x)=(1-\exp (-\lambda x))^{\alpha}$ has $\operatorname{pdf} g(x)=\alpha \lambda \exp (-\lambda x)[1-\exp (-\lambda x)]^{\alpha-1}$ which is unimodal on $(0, \infty)$ with mode at $x=(\ln \alpha) / \lambda$. Furthermore, while the exponential distribution $F$ has constant hazard rate $\lambda$, it can be shown that the exponentiated exponential (EE) $G$ has increasing hazard rate (IHD) if $\alpha>1$, constant hazard rate if $\alpha=1$ and decreasing hazard rate (DHR) if $\alpha<1$. It follows from this analysis that adding one or more parameters to a distribution makes it richer and more flexible for modeling data. Obviously $F^{\alpha}$ distributions are quite different from $F$ and need special investigations. It appears from literature that, since 1995, many researchers and authors have studied and developed various 
Review on Order Statistics and Record Values from $F^{\alpha}$ Distributions

classes of $F^{\alpha}$ distributions, among them, the exponentiated exponential and exponentiatedWeibull distributions are notable. However, in spite of the extensive recent work on $F^{\alpha}$ distributions, characterizations, estimation of parameters and their applications, very little attention has been paid to the to the study of order statistics and record values, prediction of future order statistics and record values based on existing ones, and inferential properties of order statistics and record values from $F^{\alpha}$ family of distributions. Therefore, the purpose of this research project was to study some of the distributional properties of order statistics and record values from $F^{\alpha}$ or exponentiated family of distributions. Most of the recent works are reviewed and discussed. The expressions for the pdf, cdf and moments for certain members of $F^{\alpha}$ family of distributions and associated order statistics and record values are provided. We hope that the findings of this paper will be useful for the practitioners in various fields of studies and further enhancement of research in order statistics, record value theory and their applications. These results will also be useful in the study of reversed hazard rates. One can also study the applications of order statistics and record values in the statistical data analysis when the parent distributions belong to the $F^{\alpha}$ family of distributions. It will be useful in the characterizations of $F^{\alpha}$ distributions and quantifying information contained in observing each order statistic and record value. For future work, one can consider to develop inferential procedures for the parameters of the parent $F^{\alpha}$ distributions based on the corresponding order statistics and record value distributions. It is also of theoretical interest, in designing an experiment, to investigate and establish the relationships between the record values and the order statistics when observations are taken from the same parent $F^{\alpha}$ distributions.

\section{Acknowledgement}

The authors would like to thank the editor and referees for their useful comments and suggestions which considerably improved the presentation of the paper.

\section{References}

1. Abramowitz, M., Stegun, I. A. (1970). Handbook of Mathematical Functions, with Formulas, Graphs, and Mathematical Tables. New York: Dover.

2. Afify, W. M. (2010). On estimation of the exponentiated Pareto distribution under different sample schemes. Statistical Methodology, 7 (2), 77-83.

3. Ahsanullah, M., and Nevzorov, V. B. (2001). Ordered Random Variables. Nova Science Publishers, Inc., New York, NY.

4. Ahsanullah, M. (2004). Record Values-Theory and Applications. University Press of America, Lanham, MD.

5. Ahuja, J. C., and Nash, S. W. (1967). The generalized Gompertz- 
Verhulstfamily of distributions. Sankhya, Ser. A., Vol. 29, 141 - 156.

6. Ali, M. M., and Woo, J. (2010). Estimation of tail-probability and reliability in exponentiated Pareto case. Pak. J. Statist., 26(1), 39-47.

7. Alkasasbeh, M. R., and Raqab, M. Z (2009). Estimation of the generalized logistic distribution parameters: comparative study. Statistical Methodology, 6(3), 262-279.

8. Arnold, B.C., Balakrishnan, N., and Nagaraja, H.N. (1992). A First Course in Order Statistics. John Wiley\& Sons Inc., New York. NY.

9. Asgharzadeh, A. (2006). Point and interval estimation for a generalized logistic distribution under progressive type II censoring. Commun. Statist.Theor. Meth., 35, 1685-1702.

10. Asgharzadeh, A., and Fallah, A. (2011). Estimation and prediction for exponentiatedfamily of distributions based on records. Commun. Statist.Theor. Meth., 40, 68-83.

11. Asgharzadeh, A., and Valiollahi, R. (2010). Prediction intervals for exponentiated family of distributions based on record values. Journal of Statistical Theory and Applications,9(4), 529-548.

12. Balakrishnan, N. (1990). Approximate maximum likelihood estimation for a generalized logistic distribution. Journal of Statistical Planning and Inference, 26, 221--236.

13. Balakrishnan, N., and Leung M. Y. (1988). Order statistics from the type I generalized logistic distribution. Communications in Statistics - Simulation and Computation. Vol. 17(1), 25-50.

14. Chen, G., and Balakrishnan, N. (1995). The infeasibility of probability weighted moments estimation of some generalized distributions. Recent Advances in Life-Testing and Reliability, CRC Press, Boca Raton, FL, pp. 565--573.

15. Cho, Y-S., Kang, S-B., and Han, J-T. (2009). Theexponentiated extreme value distribution. Journal of the Korean Data \&Information Science Society, 20(4), 719-731.

16. David, H.A., and Nagaraja, H. N. (2003). Order Statistics. Third Edition, John Wiley \& Sons, Inc., New York, NY.

17. Gupta, R.C., Gupta, P.L., and Gupta, R.D. (1998). Modeling failure time data by Lehman alternatives. Commun. Statist.-Theor. Meth., 27, 887-904.

18. Gupta, R.D. and Kundu, D. (1999). Generalized exponential distribution. Austral. \& New Zealand J. Statist., 41,173-188.

19. Gupta, R.D., and Kundu, D. (2000). Generalized exponential distribution: different method of estimations. J. Statist. Comput. Simul., Vol. 00, 1-22.

20. Gupta, R.D., and Kundu, D. (2001). Exponentiated exponential family: an alternative to gamma and Weibull distributions. Biometrical J., 43, 117-130.

21. Gupta, R.D., and Kundu, D. (2007). Generalized exponential distribution: Existing results and some recent developments. J. Statist. Plann. Inference, 137 (11), 3537-3547. 
Review on Order Statistics and Record Values from $F^{\alpha}$ Distributions

22. Gusmão, F. R. S., Ortega, E. M. M., and Cordeiro, G. M. (2011). The generalized inverse Weibull distribution. Statistical Papers, 52(3), 591-619.

23. Kundu, D., and Raqab, M.Z. (2005). Generalized Rayleigh distribution: different methods of estimation. Computational Statistics and Data Analysis, 49, 187-200.

24. Lehmann, E.L. (1953). The power of rank tests. Ann. Math. Statist., 24, 28-43.

25. Lemonte, A. J., and Cordeiro, G. M. (2011). The exponentiated generalized inverse Gaussian distribution. Statistics and Probability Letters, 81, 506--517.

26. Malik, H. J. (1967). Exact moments of order statistics from a power function distribution. Skand. Aktuar., 50, 64--69.

27. Mudholkar,G.S. and Hutson, A.D. (1996). ExponentiatedWeibull family: some properties and flood data application. Commun. Statist.-Theory Meth., 25, 3050-3083.

28. Mudholkar,G.S., and Srivastava, D.K. (1993). ExponentiatedWeibull family for analyzing bathtub failure data. IEEE Trans. Rel., 42, 299-302.

29. Mudholkar, G.S., Srivastava, D.K., and Freimer, M. (1995). The exponentiatedWeibull family: a reanalysis of the bus-motor-failure data. Technometrics, 37, 436-445.

30. Nadarajah, S., and Gupta, A. K. (2005). On the moments of the exponentiatedWeibull distribution. Commun. Statist.-Theor. Meth.,, 34, 253-256.

31. Nadarajah, S., and Kotz, S. (2006). The exponentiated type distributions. ActaApplicandaeMathematicae, 92, 97-111.

32. Nadarajah, S. (2011). The exponentiated exponential distribution: a survey. AStA Advances in Statistical Analysis, 95(3), 219-251.

33. Nassar, M.N., and Eissa, F.H. (2003). On exponentiatedWeibull distribution. Commun. Statist-Theory Meth., 32,1317-1336.

34. Pal, M., Ali, M. M., and Woo, J. (2006). ExponentiatedWeibull distribution. STATISTICA, 66 (2), 139-147.

35. Persson, K., and Ryden, J. (2010). ExponentiatedGumbel distribution for estimation of return levels of significant wave height. Journal of Environmental Statistics, 1(3), 1-12.

36. Pickands, J., III (1975). Statistical inference using extreme-order statistics. Ann. Statist., 3, 119-131.

37. Raqab, M.Z. (1998). Order statistics from the Burr type $X$ model. Computers Mathematics and Applications, 36, 111-120.

38. Raqab, M.Z. (2002). Inferences for generalized exponential distribution based on records. J. Statist. Plann. Infer., 104, 339-350.

39. Raqab, M.Z., and Ahsanullah, M. (2001). Estimation of the location and scale parameters of the generalized exponential distribution based on order statistics. J. Statist. Comput. Simul., 69, 109-124. 
40. Raqab, M.Z., and Kundu, D. (2006). Burr Type X distribution: revisited. Journal of Probability and Statistical Science, 4(2), 179-193.

41. Sarhan, A. E., and Greenberg, B. G. (eds.) (1962). Contributions to Order Statistics. John Wiley \& Sons, Inc., New York, NY.

42. Shadrokh, A., and Pazira, H. (2011). Estimation of parameters of the exponentiated gamma distribution in the presence of outlier. International Journal of Academic Research, Part II, 3(1), 618-624.

43. Shawky, A. I., and Abu-Zinadah, H. H. (2008). Characterizations of the exponentiated Pareto distribution based on record values. Applied Mathematical Sciences, 2(26), 1283-1290.

44. Shawky, A. I., and Abu-Zinadah, H. H. (2009). Exponentiated Pareto distribution: different method of estimations. Int. J. Contemp. Math. Sci. 4(14), 677-693.

45. Shawky, A. I., and Bakoban, R. A. (2008). Characterizations from exponentiated gamma distribution based on record values, JSTA, 7(3), 263-278.

46. Shawky, A. I., and Bakoban, R. A. (2009). Conditional expectation of certain distributions of record values. Int. J. Contemp. Math. Sciences, 4 (2), 71-91.

47. Silva, R. B., Barreto-Souza, W., and Cordeiro, G. M. (2010). A new distribution with decreasing, increasing and upside-down bathtub failure rate. Computational Statistics and Data Analysis, 54, 935-944.

48. Sultan, K. S. (2007). Order statistics from the generalized exponential distribution and applications. Commun. Statist-Theory Meth., 36, 1409-1418.

49. Surles, J.G., and Padgett, W.J. (2001). Inference for reliability and stressstrength for a scaled Burr Type X distribution. Lifetime Data Analysis, 7, 187-200. 